\title{
Ein soziales und inklusives Quartier für Alle: zur Aktualität von Lucius Burckhardts Planungsverständnis und der Forderung nach demokratischer Teilhabe an Planungsprozessen
}

Stephanie Weiss

\section{Lucius Burckhardt als Vordenker eines partizipativen und inklusiven Planungsverständnisses}

Der Schweizer Ökonom, Soziologe, Planungs- und Architekturkritiker Lucius Burckhardt $(* 1925$, Davos, $\uparrow 2003$, Basel) wird in vorliegender Auseinandersetzung als Vordenker und Wegbereiter eines auf Partizipation und Teilhabe basierenden Planungsverständnisses vorgestellt, welches sich vorrangig an den Bedürfnissen und den Gestaltungsmöglichkeiten durch ihre Nutzer*innen auszeichnet. Machtanalytische und demokratietheoretische Fragen nach Teilhabe an Planungsprozessen und ihre Ideen und Umsetzungen in Form innovativer, partizipativer Methoden an den Schnittstellen von Soziologie, Planungstheorie, Architektur und Städtebau sowie Design- und Kunstwissenschaft machen seine wissenschaftliche Popularität in Disziplinen der Stadtforschung, der Architektur und der Kunst aus. $\mathrm{Er}$ ist in den vergangenen zwei Jahrzehnten zu einem vielfältigen Ideengeber geworden, beispielsweise als Leitfigur für den Schweizer Pavillon während der Architekturbiennale 2014 in Venedig, die ihm (gemeinsam mit Cedric Price) gewidmet wurde (vgl. Baroncelli et al. 2014).

Zwar ist Burckhardt in den oben genannten Themenbereichen der Stadt- und Urbanitätsforschung sehr populär; jedoch wurde er bislang in der Sozialgeografie, insbesondere in der Quartiersforschung, eher marginal rezipiert. Ein Grund dafür mag darin liegen, dass seine Überlegungen zum sozialen Design- und Planungsverständnis sehr offen formuliert sind. Sie enthalten sehr viele inhaltliche und methodische Anknüpfungspunkte für unterschiedliche (kreative) Disziplinen im Bereich der Planung und der Stadtforschung, stellen aber keine präzise Systematik dar, beispielsweise zu Partizipationsprozessen in der Planung. Sie sind eher als eine Art kritisches Planungsdenken zu verstehen, unter Zuhilfenahme von Methoden der Wahrnehmung und des Experiments.

Durch Burckhardts Werke zieht sich die Forderung, dass Planung und Gestaltung auf alltagsweltliche, soziale und politische Fragen Antworten finden und 
Verantwortung für die Bedürfnisse von (zukünftigen) Nutzenden übernehmen müssen. Diese Forderung bezieht er auf städtebauliche und soziale Themen, die er einer Urbanismuskritik im Sinne des Sichtbarmachens und Reflektierens über komplexe politische und professionsspezifische Zusammenhänge unterzieht. Diese Position erscheint im aktuellen Planungsdiskurs außerordentlich und wegbereitend im Hinblick auf Forderungen nach (direkt)demokratischer Einflussnahme zu sein (vgl. sublurban 2017). Die Forderung nach einer umfassenden Teilhabe aller relevanten Nutzergruppen an Planungsprozessen, an der Gestaltung der gebauten Umwelt und des eigenen Lebensumfelds stellen im internationalen Diskurs derzeit ein v. a. von Bürger*innen und zivilen Akteur*innen proklamiertes gesellschaftliches Ziel dar: „Es geht nicht mehr nur um die Partizipation des Bürgers an der Planung, sondern um den Bürger als (Ko-)Produzenten der Planung“" (Siebel 2010: 29).

In aktuellen Publikationen wird Lucius Burckhardt überwiegend machtanalytisch im Kontext urbaner Entwicklung rezipiert, so auch in der bislang umfangreichsten Sekundärpublikation, die im Jahr 2014 erschienen ist (vgl. Mäder et al. 2014). Das Denken und die Pädagogik Burckhardts nehmen darin per se eine interdisziplinäre Perspektive ein, da es ihm - in der Analyse der gegenseitigen Beeinflussung von Politik, Umwelt und Mensch - um integrierte Lösungswege anhand innovativer Methoden in der Planung ging (vgl. Sutter 2014: 33 ff.; Andritzky et al. 1981).

\section{Die soziale Dimension von Planung: Triade aus Politik, Umwelt und Mensch}

Zur analytischen Betrachtung des Raumes entwirft Burckhardt eine Triade aus Politik, Umwelt und Mensch. Das Zusammenspiel dieser drei Bereiche bestimmt die Qualität des Planungsprozesses: die Möglichkeiten der Mitgestaltung durch die Benutzenden (partizipativer Anspruch, Planungskritik durch die Nutzenden), den Gebrauchswert eines Gegenstands (gemeinschaftliche Nutzung, ökonomische Kosten) und die ökologische Herstellung eines Objekts (Herstellungsbedingungen, Rohstoffverbrauch) (vgl. Burckhardt 1995). Die Triade dient als methodologisches Gerüst der Planungskritik und als soziologisches Konstrukt zur Umsetzung von Burckhardts Forderung nach Teilhabe und Partizipation. Die Qualität eines solchen Planungsprozesses stellt ein politisches Ziel dar, dessen städtebauliche Umsetzung erst durch die kontinuierliche, prozesshafte Teilhabe aller relevanten Gruppen - Nutzenden, Akteur*innen aus Politik, Planung und Verwaltung erreicht werden kann (vgl. Burckhardt 1980).

Anhand dieser Raumbetrachtungsweise ergeben sich Bezüge zur Raumtriade des französischen Stadt- und Raumtheoretiker Henri Lefebvre (1974, 2006), der 
zwar in einem marxistischen Kontext und in einer anderen wissenschaftlichen Tradition zu verorten ist; gemeinsam ist beiden Autoren jedoch eine erstaunlich ähnliche politische Argumentation vom Recht auf Teilhabe an demokratischen Entscheidungsprozessen in der Stadtentwicklung. Zudem vertreten beide Theoretiker ein ähnliches Verständnis von Raum: Der Raum ist ein Ergebnis von Beziehungsverhältnissen, wobei sich Raum und Gesellschaft gegenseitig bedingen. Der Raum besitzt eine soziale, eine materielle, eine politische und eine symbolische Bedeutung. Über seine Analyse werden sowohl Machtverhältnisse als auch Potenziale für eine gerechtere, auf Partizipation von Betroffenen gerichtete, urbane Entwicklung (vgl. Schassmann 2014: 138) sowie konkrete politische Handlungsfelder sichtbar.

Mit dem methodologischen Konstrukt der triadischen Raumanalyse - sowohl bei Lefebvre als auch bei Burckhardt - lassen sich verschiedene Raumebenen mit einer integrativen Perspektive auf das Quartier untersuchen; vom subjektiven Erleben der Raumaneignung, den Zuschreibungen über seine Symbolik, Bedeutung und Codierbarkeit, seine materielle Ausgestaltung und Strukturierung bis zur Planung des Raumes durch Verantwortliche und ihre zugrundeliegenden Modelle, Konzepte und normativen Zuschreibungen an den Raum. Olaf Schnur spricht der lefebvreschen Triade eine holistische Perspektive auf das Quartier zu, welche die Möglichkeit bietet, die Dualität von Handlung und Struktur zu überwinden und in eine ganzheitliche Betrachtung des Raumes münden zu lassen: „Gesamtgesellschaftliche Prozesse und Institutionen, die die Stadt- und Quartiersentwicklung bestimmen, sind Teil der Triade und tragen mit zu unterschiedlichen Struktur- und Handlungsmustern auf der Quartiersebene bei“" (Schnur 2012: 468).

\section{Ein soziales und inklusives Design für Alle}

Die verbindende Funktion der Triade Burckhardts - Politik, Umwelt, Mensch stellen die soziale Dimension und die soziale Bedeutung für die Nutzenden dar:

„Design ist unsichtbar. Das beste Design einer Straßenbahn wäre, wenn sie auch nachts fährt! Wir sind nicht nur von sichtbaren Gegenständen umgeben, sondern müssen den unsichtbaren Bereich, die soziale Dimension mitgestalten“ (Burckhardt 1980: 7). Und weiter fordert Burckhardt: ,,So muss sich das Design öffnen zu einem Soziodesign: einem Nachdenken über Problemlösungen“"(Burckhardt 1981: 13).

Bereits seit den 1960er-Jahren entwickelte Burckhardt ein Designverständnis, welches sich durch die Berücksichtigung der Bedürfnisse unterschiedlicher Nutzer*innengruppen, durch das Aufgreifen der Planungskritik der Nutzenden und durch die Einfachheit, Zugänglichkeit und Nutzbarkeit aller Gegenstände, der 
Architektur und der Umwelt auszeichnet. Diese Entwicklung mündete in den 1980er-Jahren in eine Zusammenarbeit mit dem Internationalen Design Zentrum Berlin, in dem er gemeinsam mit Kolleg*innen aus Architektur, Soziologie, Design und Philosophie die Ansprüche an das „Design der Zukunft“ formulierte (vgl. Burckhardt \& IDZ Berlin 1987; Burckhardt 2012). Hieraus entstand mit dem Konzept des sozialen Designs, des Soziodesigns, ein neues Design- und Entwurfsverständnis, in welchem er Gedanken zu einer Theorie des inklusiven Designs formuliert und nach dem Alltags- und Gebrauchswert des Designs für alle Nutzenden fragt. Hierbei kommt Burckhardt auch die Rolle eines theoretischen Vordenkers für aktuelle inklusive Designkonzepte wie Design für Alle, universal design oder inclusive design zu (vgl. Weiss \& Drilling 2014; 2015; Clarkson et al. 2003). Gemeinsam ist diesen Designkonzepten, dass sie zwar aus dem Diskurs und der Geschichte der Barrierefreiheit und Inklusion für behinderte Menschen entstanden sind, aber heute für einen Paradigmenwechsel hin zu einem inklusiven Designverständnis für alle Menschen stehen. Der Perspektivenwechsel stellt die Planung und Gestaltung der von vielfältigen Nutzer*innengruppen als unzulänglich empfundenen gebauten Umwelt und aller Bereiche alltäglichen Lebens in den Mittelpunkt. Nicht mehr die Behinderung oder Beeinträchtigung steht im Fokus der Argumentation, sondern die Zugänglichkeit und Hindernisfreiheit der gebauten Umwelt sowie von Produkten und Dienstleistungen des alltäglichen Gebrauchs für alle Menschen (vgl. EIDD-Design for All Europe; Build for all 2006; Internationales Design Zentrum Berlin 2008; EU-Jahr „2010: Ein hindernisfreies Europa für Alle“).

Mithilfe partizipativer Verfahren und der Beteiligung von betroffenen Menschen am Planungs- und Gestaltungsprozess können somit der bedürfnisgerechte (Um-)Bau von Gebäuden, öffentlichen Räumen und Verkehrsmitteln sowie die Nutzung von Produkten und Dienstleistungen für den privaten Gebrauch gewährleistet werden (vgl. European Commission: European Disability Strategy 2010 2020; Bauen für Alle 2006).

Burckhardts Planungsanspruch auf die Thematik der sozialen Inklusion anzuwenden, heißt demnach, dass Inklusion mithilfe von partizipativer und gestalterischer Planung durch die Nutzenden stattfindet. Deren Ziel ist ein unsichtbares, inklusives Design, das sich durch Akzeptanz, Zugänglichkeit und Aneignungsmöglichkeiten für heterogene Nutzer*innengruppen auszeichnet. Indem das (inklusive) Design unsichtbar bleibt und nicht für eine bestimmte Anspruchsgruppe deklariert und explizit erwähnt wird (z. B. rollstuhlgerecht, barrierefrei), fördert es die Akzeptanz und Zugänglichkeit für Alle. Das Design der Zugänglichkeit zu allen Bereichen alltäglichen Lebens tritt zugunsten der Funktionalität, der Einfachheit, der Sicherheit und der Ästhetik in den Hintergrund (vgl. Weiss 2011: 94; Weiss \& Drilling 2015). Das inklusive Design bleibt unsichtbar, wenn es integraler Bestandteil der Planung wird und die Funktion, Gestaltung und Nutzbarkeit 
beispielsweise eines Gebäudes oder eines Teilraumes im Quartier sozial inklusive Rollen erfüllt, ohne als solche deklariert oder sichtbar zu werden.

Aus Burckhardts Designverständnis ergeben sich übergeordnete Bezüge zum Recht auf Teilhabe relevanter Gruppen an Planungsprozessen und zur menschenrechtlichen Bedeutung von sozialer Inklusion als Ziel eines nutzer*innenzentrierten Planungsverständnisses. Soziale Inklusion bezeichnet grundsätzlich das Prinzip der gleichberechtigten Partizipation aller Menschen unter Berücksichtigung der Menschenwürde und der Anerkennung der Verschiedenheit der Menschen, der menschlichen Vielfalt, der Chancengleichheit und der Zugänglichkeit (vgl. Degener \& Mogge-Grotjahn 2012: 65 f.; UN Convention on the Rights of Persons with Disabilities 2006). Inklusion erweitert die Diskussion und Theoriebildung gegenüber dem Diskurs zur sozialen Integration insofern, als es weniger um die Integration $^{1}$ von spezifischen Gruppen (z. B. behinderte Menschen, Asylsuchende, Migrationsbevölkerung) in bestehende gesellschaftliche Systeme geht, als vielmehr um die selbstverständliche Zugehörigkeit aller Menschen in unterschiedlichen Lebenslagen und Lebensphasen zur Gesellschaft (vgl. Bielefeldt 2009; Wansing 2015).

Für die Quartiersforschung ergeben sich aus Burckhardts Prämisse Design ist unsichtbar unmittelbare Anwendungsbezüge und Forschungsfragen im Hinblick auf die Thematik der sozialen Inklusion als sozialpolitisches und gestalterisches Handlungsfeld:

- Wie kann die soziale Bedeutung eines (Quartier-)Planungsprojekts - das unsichtbare Design - sichtbar und analysierbar gemacht werden?

- Über welche sichtbaren und unsichtbaren Barrieren verfügt ein Quartier?

- Auf welche Art und Weise kann soziale Inklusion durch das Design der Zugänglichkeit und durch die Partizipation betroffener Gruppen gefördert werden?

- Mit welchen Mitwirkungsmöglichkeiten und Entscheidungsbefugnissen werden betroffene heterogene Gruppen in der Planung und Gestaltung von Quartiersentwicklungsprojekten involviert und ausgestattet?

1 „Mit dem Integrationsbegriff werden die Prozesse der Einbindung von Individuen in das normative Gefüge der Gesellschaft ebenso bezeichnet wie die Zugehörigkeit von Individuen zum gesellschaftlichen Ganzen und/oder zu gesellschaftlichen Teilbereichen. In der Integrationsperspektive erscheinen die Übernahme, Akzeptanz und Verinnerlichung der gegebenen Werte und Normen als Voraussetzung für Integration, und diese wiederum gilt als Voraussetzung für Partizipation. Integration bedeutet, dass zwei (oder mehr) Teile zu einem Ganzen werden“ (Degener \& Mogge-Grotjahn 2012: 60). 


\section{Von der Frage nach der Planungshoheit zur Spaziergangswissenschaft}

Neben der Auseinandersetzung mit dem partizipativen Design von Planungsprozessen und der Bedeutung der Inklusion stellt Burckhardt die zentrale Frage nach der Planungshoheit. Inhaltlich wiederum nicht weit entfernt, forderte Henri Lefebvre mit dem „Recht auf Stadt“ (1968) vor allem das Recht auf die Zentralität durch den konkreten Zugang zu Orten des Austauschs, der Begegnung, des Wissens, des gesellschaftlichen Reichtums usw. Es ging ihm also gegen die Verdrängung in den diskriminierenden Raum (vgl. Lefebvre, Althaler \& Schäfer 2016) sowie „ebenso [um] den Zugang zu den politischen und strategischen Debatten über die künftigen Entwicklungspfade" (Holm \& Gebhardt 2011: 8). Diese Forderungen greift Burckhardt demokratietheoretisch und methodisch auf und stellt in den 1980er-Jahren seine programmatische Frage: „Wer plant die Planung?“

Diese war nicht nur als Machtdiskurs über die Planungshoheit formuliert, sondern wurde von ihm auch weitergedacht durch die Einführung von Methoden der Partizipation für alle an einem Planungsprozess beteiligten Gruppen. Seine Frage nach der Planungshoheit findet damit direkten Anschluss an aktuelle Forderungen nach demokratischer und zivilgesellschaftlicher Teilhabe im Planungsprozess (vgl. Weiss \& Drilling 2014): Wie wird das soziale Design zu einem integralen Bestandteil des Planungsprozesses? Und: Welche Möglichkeiten und Herausforderungen ergeben sich für Politik, Architektur und Zivilgesellschaft, wenn die soziale Dimension, die in Interaktion mit ökologischer und ökonomischer Verantwortung steht, integriert werden soll?

Eine Antwort auf Burckhardts Frage nach der Planungshoheit liegt in den von ihm begründeten partizipativen und experimentellen Methoden der Wahrnehmung in der Ausbildung von Studierenden und im Einsatz in Stadtplanungsprozessen. Burckhardts Position wird auch in seiner Tätigkeit als Hochschullehrer deutlich, bei der es ihm nicht um konkrete, standardisierte Handlungsanleitungen zum Planen, Bauen und Entwerfen für seine Studierenden ging (wie in gängigen Curricula in den 1950er- und 1960er-Jahren), sondern vielmehr um das Erfahren und Erkennen von Zusammenhängen und um Problemlösungsstrategien (vgl. Schmitz 1980: 5 ff.). Sein Verständnis von der Unterrichtspraxis deckte sich mit vielen seiner Ansprüche an die Planung, die er als offenen und unkonventionellen Prozess gestaltete.

Im Jahr 1973 folgte Burckhardt als einer der Gründungsprofessoren einem Ruf auf den Lehrstuhl für Sozioökonomie urbaner Systeme der seinerzeit neu gegründeten Gesamthochschule Kassel. ${ }^{2}$ Einhergehend mit den von der Hochschul-

2 Zuvor war Lucius Burckhardt zwischen 1962 und 1973 Lehrbeauftragter und Gastprofessor am Fachbereich Architektur der Eidgenössischen Technischen Hochschule Zürich gewesen und hatte dort das sogenannte Lehrcanapé zwischen 1970 und 1973 in einer Experimentierphase als 
leitung unterstützten innovativen und unkonventionellen Lehr- und Forschungsmöglichkeiten entwickelte Lucius Burckhardt hier die Spaziergangswissenschaft, die Promenadologie, zur Wahrnehmung und Erforschung von Landschaft, welche methodisch an den Schnittstellen von Feldforschung, teilnehmender Beobachtung, künstlerischer Aktion, Intervention und konzeptionellem Gang durch die Landschaft angesiedelt ist und ein bestimmtes Thema, eine Problemstellung und das Sichtbarmachen von (problemhaften) Zusammenhängen in den Mittelpunkt stellt. So schreibt Burckhardt:

„Die Spaziergangswissenschaft ist also ein Instrument sowohl der Sichtbarmachung bisher verborgener Teile des Environments als auch ein Instrument der Kritik der konventionellen Wahrnehmung selbst“ (Burckhardt 2011: 265). Und weiter begründet Burckhardt: „Hinschauen - das tun wir oft schon gar nicht mehr. Stadtplanung, Verkehrsplanung, Soziologie - sind es nicht Schreibtischwissenschaften? Die Spaziergangswissenschaft sucht den Ort und das Lebendige auf, versucht sich darin, das Betrachten wiederzuentdecken. Betrachten heißt, neue Blickwinkel erschließen, Sehweisen ausprobieren, Ungewohntes wahrnehmen, störende Elemente aufdecken, Fehler machen und bei sich selbst bemerken. Spaziergangswissenschaft will ein anderes Verständnis von Zeit und Raum gewinnen. Spazierengehende Menschen sind schon durch den Gebrauch ihrer Füße langsamer - und da sie gehen, weil sie dazu Lust haben, und nicht, um anzukommen, sind sie zeitlich unberechenbar. Raum sieht die Spaziergangswissenschaft als Konstrukt der Wahrnehmung - also als vieldeutig." (Kulturbeutel 1993 zit. nach Schmitz 2004: 9).

Den theoretischen Hintergrund stellt dabei Burckhardts Auseinandersetzung mit der Landschaft und seiner programmatischen Frage dar: „Warum ist Landschaft schön?" (vgl. Burckhardt 2011). Und damit verbunden: Wie wird Landschaft wahrgenommen und konstruiert, welche Unterschiede gibt es zwischen Natur und Landschaft, wie entsteht Landschaft durch die Art der Fortbewegung, also das Tempo des Spazierengehens, und wie beeinflusst der Spaziergang wiederum die Wahrnehmung der Landschaft (vgl. Bossert 2014: 140; Burckhardt 2011: 266 ff.; Bürgin \& Schoch 2015)? Darüber hinaus kann der promenadologische Spaziergang auch als Aufhebung der Bedeutung des Expertenwissens zugunsten der Wichtigkeit von Alltagswissen in der Planung gesehen werden:

Verknüpfung zwischen Soziologie und Architektur in die Ausbildung von Architekturstudierenden eingeführt (vgl. Sutter 2014: 45; Blumenthal 2010). Fragen nach der sozialen Bedeutung von Architektur und Städtebau und das Thematisieren von politischen Entwicklungen waren im Lehrcanapé zentral, der architektonische Entwurf hingegen nicht die primäre Ausbildungsmethode. Der Unterricht fand zudem nicht nur in den Hörsälen an der Hochschule statt, sondern in zwischengenutzten Gebäuden in der Zürcher Innenstadt, welche Teil der Debatte um Stadtentwicklung, Proteste und deren Politisierung waren (vgl. Sutter 2014: 46 f.). 
„Der Spaziergang hat Sprengwirkung. Plötzlich ist nicht mehr klar, was vorher selbstverständlich schien. Die Hierarchie bröckelt, und es entsteht ein wunderbar offener Raum, in welchem ein umfassender Versuch für einen sinnvolleren Umgang mit der Umwelt weitergeführt werden kann“(Blumenthal 2012: 5).

Als didaktische Methode ist der promenadologische Spaziergang dem Action Teaching nahe, das „den Unterricht vom Muff des Klassenzimmers befreien“ (Burckhardt 2011: 294) und über ein Erlebnis in der Gruppe - den geplanten Spaziergang - stereotype Ansichten verändern, dekonstruieren und zu neuen Erkenntnissen führen möchte (vgl. Stippl 2011: 92 ff.; Sutter 2014: 50; Brauner \& Weisshaar 2013). Die Künstlerin und Kunstwissenschaftlerin Hannah Stippl, welche sich in ihrer Dissertation mit den landschaftstheoretischen, gemalten Aquarellen ${ }^{3}$ von Burckhardt auseinandergesetzt hat, spricht den Spaziergängen auch eine politische Bedeutung zu: „Der promenadologische Spaziergang als Performance und Demonstration konstituiert, verändert und gestaltet den öffentlichen Raum als Wahrnehmungsraum und ist dadurch zutiefst politisch“" (Stippl 2011: 108).

\section{Burckhardts Planungsvorstellung für demokratisches Planen und Bauen}

Aus einer aktuellen Planungs-, Stadt- und Quartiersentwicklungsperspektive ist es durchaus herausfordernd, eine würdigende und kritische Einordung der vielschichtigen Gedanken, Ansprüche und Forderungen Burckhardts zu unternehmen, denn seine Schriften lassen viele Lesarten zu. Das mag ein Grund sein, weshalb er zu einem Ideenspender gerade für kreative Disziplinen, die Architektursoziologie und -philosophie und für Teile der Stadtforschung geworden ist, aber sein Werk eher marginal in klassischen universitären Disziplinen rezipiert wird. Der hier skizzierte Blick ist der auf ein an den Bedürfnissen von Nutzenden zentriertes, inklusives Planungsverständnis im Sinne der Dekonstruktion und des Sichtbarmachens, wer die Planungshoheit an Planungsentscheidungen besitzt, für dessen Vorgedanken Burckhardt herangezogen werden kann.

3 Eine Auswahl an Aquarellen von Burckhardt wurde auf der Documenta 14 im Jahr 2017 in Kassel ausgestellt. Auf der Website der Documenta 14 werden die Arbeiten von Burckhardt folgendermaßen beschrieben: „Zu sehen ist eine kleine Auswahl aus über 800 ,landschaftstheoretischen Aquarellen', die der Schweizer Universalgelehrte Lucius Burckhardt während seiner jahrzehntelangen Lehrtätigkeit als Professor für Soziologie an der Architekturfakultät der Universität Kassel anfertigte. Insgesamt ergeben diese Aquarelle so etwas wie eine, Theorie der Landschaft ${ }^{\circ}$ ausgehend von einer der Grundfragen, die Burckhardt sein ganzes Lehrerleben über begleiteten: Warum ist Landschaft schön?"“ (http://www.documenta14.de/de/artists/16162/lucius-burckhardt) [Letzter Zugriff: 08.09.2018]. 
Auf einer methodologischen Ebene liegt Burckhardts Vorreiterrolle darin, ein soziales, inklusives und demokratisches Planungsverständnis gefordert zu haben, indem er sowohl eine theoretische Begründung für ein politisches Postulat der Teilhabe von betroffenen, involvierten Nutzergruppen als auch ein Repertoire von Methoden der nutzerzentrierten Stadtwahrnehmung und -planung entwickelt hat. In diesem Sinne liegen dem demokratischen Planungsverständnis methodologische Erkenntnisse aus der Lebens- und Alltagswelt der Nutzenden zugrunde, die in den Planungsprozess fließen und zu einem Hauptmerkmal der Planung werden sollen.

In Form eines kurzen Exkurses, der über die in diesem Beitrag behandelte Thematik hinausgeht, sei noch darauf hingewiesen, dass Burckhardt diese Ansprüche an die Planung und an die Ausbildung von Studierenden der Architektur und anderer Planungsprofessionen bereits in den 1960er- und 1970er-Jahren formulierte, also lange bevor Themen der Partizipation von (zukünftigen) Nutzenden in Planungsprozessen überhaupt eine reale Bedeutung in der (europäischen) Stadtplanung erfahren haben. Interessanterweise argumentiert Burckhardt bereits schon in seiner Triade aus Politik, Umwelt und Mensch inhaltlich mit Zielen des Nachhaltigkeitsdiskurses, der erst zu Beginn der 1990er-Jahre, ausgehend vom wegweisenden Bericht der Brundtland-Konferenz (UN 1987), aufkam und einen Paradigmenwechsel von den Grenzen des Wachstums (vgl. Club of Rome 1972) hin zum Leitbild der Nachhaltigkeit als einem globalen Paradigma einläutete. Was sich aus der Planungsgeschichte und aus dem Nachhaltigkeitsdiskurs herleiten lässt, ist bei Burckhardt bereits als - selbstverständlich formulierter - Umgang mit begrenzten Ressourcen, mit der Werthaftigkeit und der Wertschöpfung eines Projekts sowie mit der Bedeutung für die Gemeinschaft in Planungsprozessen angelegt (vgl. Burckhardt \& Brock 1985; Weiss \& Drilling 2014).

Diese bereits vor über 40 Jahren formulierten Ansprüche korrespondieren mit heutigen Nachhaltigkeitszielen und sind anschlussfähig an Fragen nach Teilhabe und Partizipation im aktuellen Diskurs an nachhaltiger und inklusiver Stadt- und Quartiersentwicklung, insbesondere was den Einbezug von Nutzenden und die Bedeutung, die ein Planungsvorhaben im Alltag der Stadt- und Quartiersbevölkerung einnimmt, angeht (vgl. Weiss 2015; Drilling \& Weiss 2012). Burckhardts Planungsanspruch liegt in einer partizipativen Grundhaltung, deren Betrachtungsweisen vom jeweiligen Ort ausgehen und denen die Betrachter*innen durch ihre Wahrnehmungen und Zuschreibungen Sinn verleihen, der aus der Unmittelbarkeit heraus entsteht, der aber auch als wichtiges und zu reflektierendes Kriterium in die Planung fließen soll.

An den Schnittstellen zwischen (urbaner) Ökologie, Partizipation vielfältiger Gruppen und mithilfe performativer und partizipativer Methoden steht Burckhardts Planungsverständnis wegbereitend für aktuelle und populäre Themen, 
Aktionen und Forderungen nach Teilhabe an (direkt)demokratischen Stadt-, Raum- und Quartiersentwicklungsprozessen (vgl. beispielhaft Harvey 2013; Mullis 2014; HABITAT III).

\section{Literatur}

Andritzky, M., Burckhardt, L. \& Hoffmann, O. (Hrsg.) (1981): Für eine andere Architektur. Eine Publikation des Deutschen Werkbundes. 2 Bände. Frankfurt am Main: FischerTaschenbuch-Verlag.

Baroncelli, L. et al. (2014): Lucius Burckhardt \& Cedric Price - a stroll through a fun palace: Swiss Pavilion, Biennale Architettura 2014. Zürich: Swiss Arts Council Pro Helvetia.

Build for all Projektpartner (Hrsg.) (2006): Bauen für Alle. Förderung der Zugänglichkeit für Alle in der baulichen Umwelt und öffentlichen Infrastruktur. Leitfaden. Gefördert durch die Europäische Kommission. Dokument zur öffentlichen Beratung. Luxemburg.

Becker, E. et al. (Hrsg.) (2010): Stadtentwicklung, Zivilgesellschaft und bürgerschaftliches Engagement. Berlin, Boston: De Gruyter.

Bielefeldt, H. (2009): Zum Innovationspotenzial der UN-Behindertenrechtskonvention. Essay No. 5, 3. aktualisierte und erweiterte Auflage. Deutsches Institut für Menschenrechte (Hrsg.). Berlin.

Blumenthal, S. (2010). Das Lehrcanapé: Lucius Burckhardt und das Architektenbild an der ETH Zürich 1970-1973 (Vol. No. 2, Standpunkte Dokumente). Basel: Standpunkte.

Blumenthal, S. (2012): Vorwort. In: Burckhardt, Lucius: Design ist unsichtbar. Entwurf, Gesellschaft und Pädagogik. Blumenthal, S. et al. (Hrsg.). Berlin: Martin Schmitz Verlag, 5-11.

Bossert, M. (2014): Spazieren als Wissenschaft. In: Mäder, Ueli et al. (Hrsg.): Raum und Macht: Die Stadt zwischen Vision und Wirklichkeit: Leben und Wirken von Lucius und Annemarie Burckhardt. Zürich: Rotpunktverlag: 139-154.

Brauner, M. \& Weisshaar, B. (Hrsg.) (2013): Spaziergangswissenschaft in Praxis: Formate in Fortbewegung. Berlin: Jovis.

Bürgin, R. \& Schoch, A. (2015): Wie Spazieren Wissen schafft: Über die Spaziergangswissenschaft nach Lucius und Annemarie Burckhardt. In: Archithese 2: 72-79.

Burckhardt, L. (1980): Wer plant die Planung? Architektur, Politik, Mensch. Fezer, J.; Schmit, M. (Hrsg.). Kassel: Martin Schmitz Verlag.

Burckhardt, L. (1981): Design ist unsichtbar. In: Gsöllpointer, H., Hareiter, A. \& Ortner, L. (Hrsg.): Design ist unsichtbar. Publikation anlässlich der Ausstellung Forum Design, Linz, 27. Juni bis 5. Oktober 1980. Wien: LV Löcker Verlag: 13-20.

Burckhardt, L. \& Brock, B. (1985): Die Kinder fressen ihre Revolution: Wohnen - Planen - Bauen - Grünen. Köln: DuMont.

Burckhardt, L. (1995): Design = unsichtbar. Höger, H. (Hrsg.). Ostfildern: Hatje Cantz.

Burckhardt, L. (2011): Warum ist Landschaft schön? Die Spaziergangswissenschaft. Ritter, M.; Schmitz, M. (Hrsg.). Berlin: Martin Schmitz Verlag. 
Burckhardt, L. (2012): Design ist unsichtbar. Entwurf, Gesellschaft und Pädagogik. Blumenthal, S. et al. (Hrsg.). Berlin: Martin Schmitz Verlag.

Burckhardt, L. \& Internationales Design Zentrum Berlin (1987): Design der Zukunft. Architektur. Technik. Ökologie. Berlin: Dumont.

Clarkson, J. et al. (Hrsg.) (2003): Inclusive Design: Design for the whole population. Springer: London.

Club of Rome (1972): Die Grenzen des Wachstums: Bericht des Club of Rome zur Lage der Menschheit (DVA Informativ). Stuttgart: Deutsche Verlags-Anstalt. Abrufbar unter: http:/www.donellameadows.org/wp-content/userfiles/Limits-to-Growth-digitalscan-version.pdf. Letzter Zugriff: 8.9.2018.

Degener, T. \& Mogge-Grotjahn, H. (2012): „All inclusive“? Annäherungen an ein interdisziplinäres Verständnis von Inklusion. In: Balz, H.-J., Benz, B. \& Kuhlmann, C. (Hrsg.): Soziale Inklusion. Grundlagen, Strategien und Projekte in der Sozialen Arbeit. Wiesbaden: Springer Fachmedien, 59-77.

Drilling, M. \& Weiss, S. (2012): Soziale Nachhaltigkeit in der Siedlungsentwicklung. Raum \& Umwelt: Schriftenreihe der Schweizerischen Vereinigung für Landesplanung VLPASPAN, Bern.

EIDD - Design for All Europe Stockholm Declaration 2004. Adopted on 9 May 2004 at the Annual General Meeting of the European Institute for Design and Disability in Stockholm. Abrufbar unter: http://dfaeurope.eu/what-is-dfa/dfa-documents/the-eiddstockholm-declaration-2004. Letzter Zugriff: 7.7.2017.

European Commission: European Disability Strategy 2010-2020: A Renewed Commitment to a Barrier-Free Europe Initial plan to implement the European Disability Strategy 2010-2020. Abrufbar unter: http://eur-lex.europa.eu/LexUriServ/LexUriServ. do?uri=COM:2010:0636:FIN:en:PDF. Letzter Zugriff: 8.9.2018.

Harvey, D. (2013): Rebellische Städte: Vom Recht auf Stadt zur urbanen Revolution. Berlin: Suhrkamp.

Gsöllpointer, H., Hareiter, A. \& Ortner, L. (Hrsg.) (1980): Design ist unsichtbar. Publikation anlässlich der Ausstellung Forum Design, Linz, 27. Juni bis 5. Oktober 1980. Wien: LV Löcker Verlag.

Habitat III - 2016: The United Nations Conference on Housing and Sustainable Urban Development. Abrufbar unter: http://habitat3.org. Letzter Zugriff: 8.9.2018.

Holm, A. \& Gebhardt, D. (2011): Initiativen für ein Recht auf Stadt. Theorie und Praxis städtischer Aneignung. Hamburg: VSA Verlag.

Internationales Design Zentrum Berlin e. V. (Hrsg.) (2008): Universal Design. Gefördert vom Bundesministerium für Familie, Senioren, Frauen und Jugend im Rahmen der Initiative Wirtschaftsfaktor Alter. Berlin.

Lefebvre, H. (1968): Le droit à la ville (Société et urbanisme). Paris: Éditions anthropos.

Lefebvre, H. (1974): La production de l'espace. Paris: Éditions anthropos.

Lefebvre, H. (2006): Die Produktion des Raumes, In: Dünne, J. \& Günzel, S. (Hrsg.): Raumtheorie. Grundlagentexte aus Philosophie und Kulturwissenschaften, Frankfurt am Main, 330-340.

Lefebvre, H., Althaler, B. \& Schäfer, C. (2016): Das Recht auf Stadt (Deutsche Erstausgabe, 1. Auflage ed., Nautilus Flugschrift). Hamburg: Edition Nautilus. 
Mäder, U. et al. (Hrsg.) (2014): Raum und Macht: Die Stadt zwischen Vision und Wirklichkeit: Leben und Wirken von Lucius und Annemarie Burckhardt. Zürich: Rotpunktverlag.

Mullis, D. (2014): Recht auf die Stadt: Von Selbstverwaltung und radikaler Demokratie. Münster: Unrast.

Schassmann, H. (2014): Henri Lefebvre. In: Mäder, U. et al. (Hrsg.): Raum und Macht: Die Stadt zwischen Vision und Wirklichkeit: Leben und Wirken von Lucius und Annemarie Burckhardt. Zürich: Rotpunktverlag, 123-138.

Schmitz, M. (1980): Von der Urbanismuskritik zur Spaziergangswissenschaft. In: Burckhardt, L.: Wer plant die Planung? Architektur, Politik und Mensch. Kassel: Martin Schmitz Verlag, 5-10.

Schmitz, M. (2004): Von der Urbanismuskritik zur Spaziergangswissenschaft. In: Burckhardt, L.: Wer plant die Planung? Architektur, Politik und Mensch. Kassel: Martin Schmitz Verlag, 5-10.

Schnur, O. (2012): Quartier und Nachbarschaft. In: Eckhardt, F. (Hrsg.): Handbuch Stadtsoziologie. Wiesbaden: VS Verlag, 449-474.

Siebel, W. (2010): Planende Verwaltung und zivile Gesellschaft. In: Becker, E. et al. (Hrsg.): Stadtentwicklung, Zivilgesellschaft und bürgerschaftliches Engagement. Berlin, Boston: De Gruyter, 25-38.

Stippl, H. (2011): Nur wo der Mensch die Natur stört, wird die Landschaft wirklich schön. Die landschaftstheoretischen Aquarelle von Lucius Burckhardt. Dissertation Wien.

sublurban: Zeitschrift für kritische Stadtforschung. Band 5, 1/2017. Wer plant die Planung? Lucius Burckhardt, kommentiert von W. Reuter, B. Binder, J. S. Dangschat, M. Grubbauer, M. Guggenheim, Stadt von unten, I. Dzudzek, 105-171.

Sutter, P. (2014): Lucius Burckhardt-Wackernagel. In: Mäder, U. et al. (Hrsg.): Raum und Macht: Die Stadt zwischen Vision und Wirklichkeit: Leben und Wirken von Lucius und Annemarie Burckhardt. Zürich: Rotpunktverlag, 21-64.

UN (1987): Report of the World Commission on Environment and Development: Our Common Future. Abrufbar unter: www.un-documents.net/wced-ocf.htm. Letzter Zugriff: 8.9.2018.

UN (2006): Convention on the Rights of Persons with Disabilities. Abrufbar unter: https://www.un.org/development/desa/disabilities/convention-on-the-rights-of-personswith-disabilities.html. Letzter Zugriff: 9.9.2018.

Wansing, G. (2015): Was bedeutet Inklusion? Annäherungen an einen vielschichtigen Begriff. In: Degener, T. \& Diehl, E. (Hrsg.): Handbuch Behindertenrechtskonvention: Teilhabe als Menschenrecht - Inklusion als gesellschaftliche Aufgabe (Vol. Bd. 1506, Schriftenreihe/Bundeszentrale für Politische Bildung). Bonn: BpB, 43-54.

Weiss, S. (2011): „Design für Alle“ als Gestaltungsparadigma der Zugänglichkeit. Eine Diskussion zu Möglichkeiten und Grenzen sozialer Inklusion durch das Design öffentlicher Räume. In: Emmenegger, B. \& Litscher, M. (Hrsg.): Perspektiven zu öffentlichen Räumen. Theoretische und praxisbezogene Beiträge aus der Stadtforschung. Luzern: interact-Verlag, 85-103.

Weiss, S. (2015): Das Konzept „Design für Alle“ und die Bedeutung der Hindernisfreiheit in Wohnumfeld und Quartier. In: Neuhaus, F., Schumacher, C. \& Selva, L. (Hrsg.): 
Hindernisfreies Wohnen: Mobilisierungspotential des Gebäudebestandes am Beispiel des Kantons Basel Stadt. Muttenz: FHNW, 52-54.

Weiss, S. \& Drilling, M. (2014): Soziale Arbeit: Partizipative Planung. In: Mäder, U. et al. (Hrsg.): Raum und Macht: Die Stadt zwischen Vision und Wirklichkeit: Leben und Wirken von Lucius und Annemarie Burckhardt. Zürich: Rotpunktverlag, 119-121.

Weiss, S. \& Drilling, M. (2015): Die Stadt, der Mensch und das Design. Zum sozialen Planungsverständnis von Lucius Burckhardt. In: Archithese 2/2015, 96-101.

Open Access Dieses Kapitel wird unter der Creative Commons Namensnennung 4.0 International Lizenz (http://creativecommons.org/licenses/by/4.0/deed.de) veröffentlicht, welche die Nutzung, Vervielfältigung, Bearbeitung, Verbreitung und Wiedergabe in jeglichem Medium und Format erlaubt, sofern Sie den/die ursprünglichen Autor(en) und die Quelle ordnungsgemäß nennen, einen Link zur Creative Commons Lizenz beifügen und angeben, ob Änderungen vorgenommen wurden.

Die in diesem Kapitel enthaltenen Bilder und sonstiges Drittmaterial unterliegen ebenfalls der genannten Creative Commons Lizenz, sofern sich aus der Abbildungslegende nichts anderes ergibt. Sofern das betreffende Material nicht unter der genannten Creative Commons Lizenz steht und die betreffende Handlung nicht nach gesetzlichen Vorschriften erlaubt ist, ist für die oben aufgeführten Weiterverwendungen des Materials die Einwilligung des jeweiligen Rechteinhabers einzuholen.

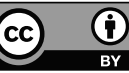

